This item was submitted to Loughborough's Research Repository by the author.

Items in Figshare are protected by copyright, with all rights reserved, unless otherwise indicated.

\title{
On a switching control scheme for nonlinear systems with ill-defined relative
} degree

PLEASE CITE THE PUBLISHED VERSION

PUBLISHER

(c) Elsevier

LICENCE

CC BY-NC-ND 4.0

REPOSITORY RECORD

Chen, Wen-Hua. 2008. "On a Switching Control Scheme for Nonlinear Systems with III-defined Relative Degree”. figshare. https://hdl.handle.net/2134/3797. 
This item was submitted to Loughborough's Institutional Repository (https://dspace.lboro.ac.uk/) by the author and is made available under the following Creative Commons Licence conditions.

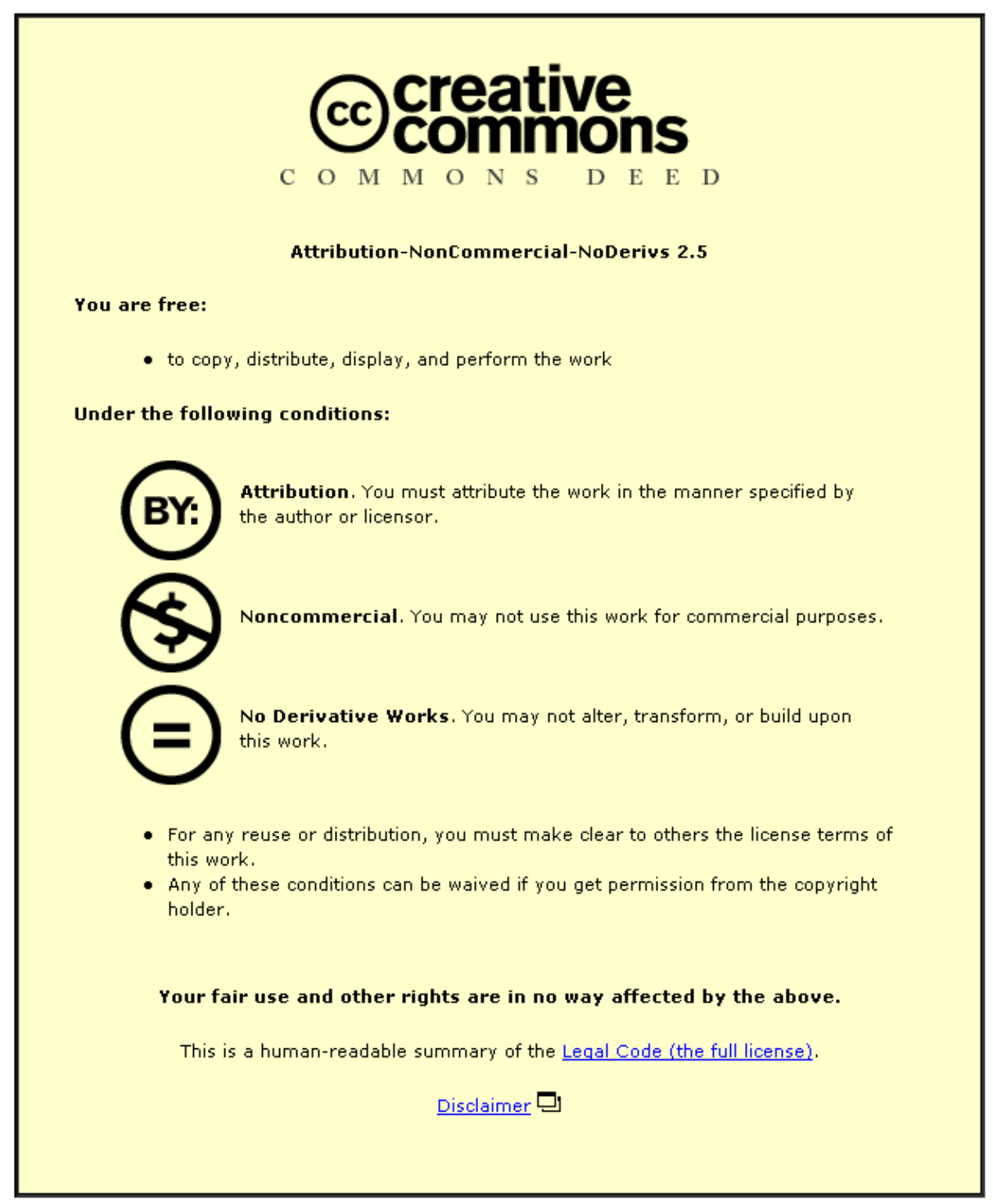

For the full text of this licence, please go to: http://creativecommons.org/licenses/by-nc-nd/2.5/ 


\title{
On a Switching Control Scheme for Nonlinear Systems with Ill-Defined Relative Degree
}

\author{
Wen-Hua Chen ${ }^{\mathrm{a}}$ and Donald J. Ballance ${ }^{\mathrm{b}}$ \\ ${ }^{a}$ Department of Aeronautical and Automotive Engineering \\ Loughborough University \\ Loughborough, Leicestershire LE11 3TU, UK. \\ Email:w.chen@elec.gla.ac.uk \\ ${ }^{\mathrm{b}}$ Centre for Systems $\&$ Control \\ and Department of Mechanical Engineering \\ University of Glasgow \\ Glasgow G12 8QQ, UK. \\ Email: D.Ballance@mech.gla.ac.uk
}

\begin{abstract}
This paper discusses the applicability of a switching control scheme for a nonlinear system with ill-defined relative degree. The control scheme switches between exact and approximate input-output linearisation control laws. Unlike a linear system under a switching control scheme, the equilibria of a nonlinear system may change with the switching. It is pointed out that this is not sufficient to cause instability. When the region of the approximate linearisation control law is attractive to the exact zero dynamics, it is possible that the closed-loop system under the switching control scheme is still stable. The results in this paper shows that the switching control scheme proposed in Tonlin and Sastry (Systems \& Control Letters 35(3)(1998) 145-154) is applicable for a wider class of nonlinear systems.
\end{abstract}

Key words: Switching, Nonlinear control, Zero dynamics, Hybrid systems, Stability 


\section{Introduction}

Geometric nonlinear theory has become one of the most promising design tools for nonlinear systems in the past decade. However one of the main assumptions in the feedback linearisation technique is that the nonlinear system under consideration should have well defined relative degree. For those systems with ill-defined relative degree, the input-output linearising law has singularities. This restricts the application of feedback linearisation technique in many engineering systems. One of the main approaches to this problem is to use a switching control scheme [4], that is, a tracking control law that switches between an approximate input-output linearisation control law when the state is close to the singularities and an exact input-output linearisation control law when the state is far from the singularities. However, in some cases, this scheme does not work well. For example, when such a scheme is applied in the ball and beam example [2], the flip behaviour appears and the beam cyclically oscillates between 0 and $\pi$ [4]. This motivates research on the applicability of this approach. On the other hand, when a switching control scheme is involved in a nonlinear system, the whole system is a kind of hybrid system. The study of hybrid systems is currently an active research area. It is interesting to understand the behaviour of this hybrid system and investigate the applicability of the switching control scheme. In contrast to switching control for linear systems, the equilibria of nonlinear systems may change under switching control schemes. The applicability of this switching linearisation approach was assessed by considering the behaviour of the system's zero dynamics at the switching boundary [4]. This paper further investigates the applicability of the switching linearisation control scheme. It is pointed out that the switching control scheme proposed by Tomlin and Sastry [4] is applicable not only for nonlinear system having unchanged equilibria but also for nonlinear system whose equilibria change under the switching control. It is shown by two examples that the nonlinear systems can still be stable even though a change in the equilibria between the exact and approximate internal dynamics occurs. The necessary condition for the switching control scheme to be stable is that the region for the approximate feedback linearisation is attractive to the closed-loop system under the exact linearisation control law. 


\section{Main Results}

\subsection{Switching control scheme}

Consider a single input and single output affine nonlinear system

$$
\left\{\begin{array}{l}
\dot{x}=f(x)+g(x) u \\
y=h(x)
\end{array}\right.
$$

where $x \in R^{n}, u \in R$ and $y \in R$ are the state vector, input and output respectively. $f(x)$ and $g(x)$ are analytic function vector fields and $h(x)$ is an analytic function. It is also supposed that the controllability rank condition $\operatorname{rank}\left\{g, a d_{f} g, \ldots, a d_{f}^{n-1} g\right\}=n$ holds for the system (1). If $x_{s}$ is a point such that $L_{g} L_{f}^{r-1} h(x)=0$ where $r$ is the relative degree of the nonlinear system (1), then $x_{s}$ is called a singular point of the nonlinear system (1). A nonlinear system with such a singular point is known as a nonlinear system with ill-defined relative degree. Define the set of all singular points

$$
N_{s}=\left\{x \in R^{n} \mid L_{g} L f^{r-1} h(x)=0\right\}
$$

Let $x_{0}$ be an equilibrium of the system (1), that is, $f\left(x_{0}\right)=0$. Without loss of generality, it is assumed that $x_{0}$ belongs to the set $N_{s}$ and $h\left(x_{0}\right)=0$ [4]. That is, the equilibrium point $x_{0}$ is a singular point.

Based on the approximate linearisation method for nonlinear systems with ill-defined relative degree [2], Tomlin and Sastry propose a switching control scheme where an approximate linearisation control law is used when the state is close to the singular points and an exact linearisation control law is employed when the state is far away from the singular points [4]. This scheme has strengths from both approximate and exact linearisation techniques. However, since the switching is involved, poor performance may result when this scheme is applied to some nonlinear systems [4].

Let

$$
M_{0}=\left\{x \in R^{n}: L_{g} L_{f}^{r-1} h(x) \leq \delta\right\}
$$


and

$$
M=\left\{x \in R^{n}: L_{g} L_{f}^{r-1} h(x)>\delta\right\}
$$

where $\delta$ is a positive scalar. Then the closed-loop system under the proposed switching control scheme is given by

$$
\left\{\begin{array}{l}
\dot{x}=f^{\mathrm{ex}}\left(x, y_{D}\right)=f(x)+g(x) u^{\mathrm{ex}}\left(x, y_{D}\right) \quad x \in M \\
\dot{x}=f^{\mathrm{app}}\left(x, y_{D}\right)=f(x)+g(x) u^{\mathrm{app}}\left(x, y_{D}\right) x \in M_{0}
\end{array}\right.
$$

where $u^{\text {ex }}$ is the exact linearisation control law; for example see [3], and $u^{\text {ex }}$ is the approximate linearisation control law in [2]. $y_{D}$ denotes the reference signal.

The internal dynamics can be divided into two cases: zero dynamics $\left(y_{D}=0\right)$ and the driven dynamics $\left(y_{D} \neq 0\right)$. For sake of simplicity, only the zero dynamics are considered first but the driven dynamics can also be investigated by the same method [4] and will be illustrated by an example. In what follows, the zero dynamics of the closed-loop system under the exact and approximate linearisation control laws are referred to as exact zero dynamics and approximate zero dynamics respectively. $x_{0}$ is always the equilibrium of the approximate zero dynamics when the approximate input-output linearisation control law is appropriately designed. But due to the singularity, the exact zero dynamics are divided into the following three cases [4]:

Case 1: Exact zero dynamics do not exist since there does not exist any input that will hold the output and its derivatives at zero;

Case 2: Exact zero dynamics exist but $x_{0}$ is not an equilibrium;

Case 3: Exact zero dynamics exist and $x_{0}$ is an equilibrium.

Case 3 where equilibria remain unchanged under the switching has been carefully studied in [4]. It is shown that the switching control scheme could work well in this case. When the control scheme switching between the exact and approximate linearisation control laws is designed and implemented on the systems belonging to the Case 1 and 2, a change of equilibrium occurs. Since it is believed [4] that the change in the equilibrium results in instability, one may conclude that a control scheme which switches between exact and approximate control laws is not likely to work for these cases. This paper adds to the results 
in [4] and further investigates the applicability of the switching control scheme in Cases 1 and 2. We start from the following examples and show that a change of equilibrium is not sufficient to cause instability.

\subsection{Example 1: Case 1}

Consider a nonlinear system (1) with

$$
\begin{gathered}
x=\left[x_{1}, x_{2}, x_{3}\right]^{T} ; \\
f(x)=\left(\begin{array}{c}
-x_{2} \\
4 x_{1} \\
x_{2}
\end{array}\right) ; \\
g(x)=\left(\begin{array}{c}
1 \\
x_{1}^{2} \\
0
\end{array}\right) ;
\end{gathered}
$$

and

$$
h(x)=x_{3}
$$

For this system we have

$$
\begin{gathered}
\frac{\partial h}{\partial x}=(0,0,1) \\
L_{g} h(x)=0 ; \quad L_{f} h(x)=x_{2} \\
\frac{\partial L_{g} h(x)}{\partial x}=(0,1,0) \\
L_{g} L_{f} h(x)=x_{1}^{2} ; \quad L_{f}^{2} h(x)=4 x_{1}
\end{gathered}
$$

Thus the system has a relative degree 2 when $x_{1} \neq 0$. It is singular at $x_{1}=0$. The relative degree of this nonlinear system is not well-defined. 
It follows from (13) that

$$
\begin{gathered}
\frac{\partial L_{f}^{2} h(x)}{\partial x}=(4,0,0) \\
L_{g} L_{f}^{2} h(x)=4 ; \quad L_{f}^{3} h(x)=-4 x_{2}
\end{gathered}
$$

According to the robust relative degree definition in [4], the system has robust relative degree 3 and thus the difference between the robust relative degree and the relative degree is 1 .

It is also easy to check that the origin $(0,0,0)$ is the unique equilibrium point for this system, which is included in the set of singular points $N_{s}=\left\{x \in R^{3}: x_{1}=0\right\}$.

The exact input-output linearisation control law can be given by

$$
u^{\mathrm{ex}}(x)=\frac{-4 x_{1}+v}{x_{1}^{2}}
$$

where

$$
v=\ddot{y}_{D}+\alpha_{2}\left(\dot{y}_{D}-x_{2}\right)+\alpha_{1}\left(y_{D}-x_{3}\right)
$$

Similar to [4], the approximate linearisation control law can be given by

$$
u^{\mathrm{app}}(x)=x_{2}+v / 4
$$

where

$$
v=y_{D}^{(3)}+\alpha_{3}\left(\ddot{y}_{D}-4 x_{1}\right)+\alpha_{2}\left(\dot{y}_{D}-x_{2}\right)+\alpha_{1}\left(y_{D}-x_{3}\right)
$$

Since $L_{g} L_{f} h(x)=x_{1}^{2}$ and $L_{f}^{2} h(x)=4 x_{1}$, this is exactly Case 1 [4]. A control scheme switching between (16) and (18) can work for the nonlinear system (1) with (7), (8) and (9) although a change of equilibrium occurs. To show this, we choose $\left(\alpha_{1}, \alpha_{2}\right)$ in $(17)$ and $\left(\alpha_{1}, \alpha_{2}, \alpha_{3}\right)$ in $(19)$ as $(1,2)$ and $(1,3,3)$, respectively. The parameter $\delta$ is chosen as 0.1 . That is, the exact linearisation control law (16-17) and the approximate linearisation control law (18-19) work in the regions

$$
M=\left\{x \in R^{3}:\left|x_{1}\right|>0.1\right\}
$$


and

$$
M_{0}=\left\{x \in R^{3}:\left|x_{1}\right| \leq 0.1\right\}
$$

respectively.

For simplicity, let the tracking signal $y_{D}$ be zero and then the driven dynamics are the same as the zero dynamics. Moreover, the desired state is the origin in this case, which is a singular point. When the initial state is $(2,-2,2)$, the response under the switching control is depicted in Fig. 1. It is shown that the control scheme which switches between the exact and the approximate linearisation control laws (16) and (18) works very well for this example.

To show that this switching control scheme can track reference signals whose magnitude and derivatives are not small, the reference signal in the ball and beam example in [4] is adopted, that is, $y_{D}=1.9 \sin (1.3 t)+3$. The simulation results in Fig. 2 show that the plant can track this reference signal well under this switching scheme. The dash-dot and solid lines are the histories of the reference signal and the system's output respectively. The driven dynamics is shown by the history of the state $x_{1}$. It should be noted that the control scheme switches between the exact and approximate control laws depending on the state $x_{1}$, which keeps going through the switching boundaries.

\subsection{Example 2: Case 2}

The system to be considered is given by (1) with $h(x)$ given in (9) and

$$
\begin{gathered}
f(x)=\left(\begin{array}{c}
-x_{2}-x_{1} \\
x_{1} \\
x_{2}
\end{array}\right) ; \\
g(x)=\left(\begin{array}{c}
0.05 \\
x_{1} \\
0
\end{array}\right)
\end{gathered}
$$


Following the same procedure as in Example 1, we have

$$
L_{g} L_{f} h(x)=x_{1} ; \quad L_{f}^{2} h(x)=x_{1} .
$$

Since $L_{g} L_{f} h(x)$ and $L_{f}^{2} h(x)$ are the function of $x_{1}$ with the same order, this is exactly Case 2 as discussed in [4].

Similarly, the exact and approximate linearisation control laws can be given by

$$
\begin{gathered}
u^{\mathrm{ex}}(x)=\frac{-x_{1}+v}{x_{1}} ; \\
v=\ddot{y}_{D}+\alpha_{2}\left(\dot{y}_{D}-x_{2}\right)+\alpha_{1}\left(y_{D}-x_{3}\right)
\end{gathered}
$$

and

$$
\begin{gathered}
u^{\mathrm{app}}(x)=\left(x_{1}+x_{2}+v\right) / 0.05 \\
v=y_{D}^{(3)}+\alpha_{3}\left(\ddot{y}_{D}-x_{1}\right)+\alpha_{2}\left(\dot{y}_{D}-x_{2}\right)+\alpha_{1}\left(y_{D}-x_{3}\right)
\end{gathered}
$$

respectively. It can be shown that

$$
\lim _{x_{1} \rightarrow 0} u_{0}^{\mathrm{ex}}(x)=-1
$$

which is not equal to zero and less than infinity. The origin is not an equilibrium of the exact zero dynamics.

The simulation result with the same design parameters and simulation conditions as in Example 1 is plotted in Fig. 3. The simulation shows that such a switching control strategy also works well for this system.

\subsection{Discussion}

Although the change in the equilibrium of the zero dynamics does not imply that the closed-loop system under the switching control scheme is unstable, the behaviour of the zero dynamics does play an important role in stability of the system under the switching control scheme. 
Consider Example 1. Although the exact zero dynamics do not exist, the region $M_{0}$ is attractive to the "exact zero dynamics", that is, the exact linearisation control law drives the state to the approximate region $M_{0}$. Once the state arrives in the region $M_{0}$, the approximate linearisation control law steers the state to the origin. This is the same as in Example 2. In Example 2, the exact zero dynamics are given by

$$
\dot{x}_{1}=x_{1}-0.05 \text {. }
$$

Thus the equilibrium of the exact zero dynamics is $x_{1}=0.05$, which belongs to the set $M_{0}$. That is, the region $M_{0}$ is attractive to the exact zero dynamics.

For a regulation problem, the necessary condition for the closed-loop nonlinear system under the switching control scheme to be stable is that the approximate region $M_{0}$ must be an attractive region of the "exact zero dynamics". This can be proved by contradiction. The change in the equilibrium happens only for Cases 1 and 2. Thus Case 3 does not need to be considered. First consider Case 2. When the region $M_{0}$ is not attractive to the exact zero dynamics, it implies the exact zero dynamics has at least one equilibrium in the region $\mathrm{M}^{+}$ or $M^{-}$. The state starts from the region near around the equilibrium will be attracted to the equilibrium and remain on this equilibrium under the exact input-output linearisation control law. It is impossible to arrive at the desired point $x_{0}$, which belongs to the set $M_{0}$,

by the switching control scheme. Similarly, one can show the same result holds for Case 1 since the region $M_{0}$ is not attractive to all the states under the exact linearisation control law.

The stability analysis of a general tracking problem for a nonlinear system under the proposed switching control scheme is much more complicated. However, the above condition still holds since a regulation problem can be considered as a special case of the tracking problem where the tracking reference is zero. We will discuss this in the next section using the ball and beam example.

\section{The ball and beam example revisited}

The ball and beam example motivated the study of the applicability of the switching control scheme [4]. Now we re-visit this example. 
The ball and beam example is described by

$$
\left\{\begin{array}{l}
\dot{x}_{1}=x_{2} \\
\dot{x}_{2}=B\left(x_{1} x_{4}^{2}-G \sin x_{3}\right) \\
\dot{x}_{3}=x_{4} \\
\dot{x}_{4}=u
\end{array}\right.
$$

and the output equation is

$$
y=x_{1}-r_{0}
$$

where $x_{1}, x_{2}, x_{3}$ and $x_{4}$ are the ball position, ball velocity, beam angle and beam velocity respectively, and $r_{0}$ is the offset constant.

It is easy to show that the exact input-output linearisation is not defined when $x_{1} x_{4}=0$. Hence the set of the singular points is given by

$$
N_{s}=\left\{x \in R^{4}: x_{1} x_{4}=0\right\}
$$

Following the switching control scheme in Section 2.1, the state space can be partitioned as

$$
\begin{aligned}
& M_{0}=\left\{x \in R^{4}:\left|x_{1} x_{4}\right| \leq \delta\right\} \\
& M^{+}=\left\{x \in R^{4}: x_{1} x_{4}>\delta\right\}
\end{aligned}
$$

and

$$
M^{-}=\left\{x \in R^{4}: x_{1} x_{4}<-\delta\right\}
$$

where $M=M^{+} \cup M^{-}$. When the state enters the region $M_{0}$, the approximate feedback linearisation control law is employed and when the state is outside the region $M_{0}$, the exact feedback linearisation control law is used. As shown by [4], although the control scheme which switches between the exact and approximate linearisation control laws is stable, the driven dynamics cause the beam to continuously flip upside down and back again (see Figure 4 which is reproduced from Figure 2 in [4] for the convenience of the reader). The ball and beam plant belongs to Case 2 . 
This paper shows that the flip behaviour in the ball and beam example is mainly due to its unstable zero dynamics rather than the switching control scheme. In fact, as shown in [4], the zero dynamics under the exact linearisation control law are one dimensional

$$
\dot{x}_{4}=G \cos x_{3} / 2 r_{0} \text { where } r_{0} x_{4}^{2}=G \sin x_{3},
$$

which is shown in Figure 4. As shown in Figure 4, $x_{1}$ is far from zero in the simulation where $y_{D}=1.9 \sin (1.3 t)+3$. The singularities are due to the state $x_{4}$ going through zero. The set $M_{0}$ is shown in Figure 5 in the $x_{3}-x_{4}$ state-plane.

The flip behaviour can be explained as follows: When the reference $y_{D}$ is tracked, all the states vary due to the internal driven dynamics. In the beginning, the state $x_{1}$ is small, and $x_{4}$ is within the set $M_{0}$. According to the switching control scheme, the ball and beam example is now controlled by the approximate linearisation control law [2]. Since $\left(r_{0}, 0,0,0\right)$ is one of the two equilibria under the approximate linearisation control law, $x_{3}$ varies around 0 due to the driven internal dynamics. It should be noted that the switching boundary for $x_{4}$ depends on the magnitude of $x_{1}$. When $x_{1}$ increases such that the magnitude of $x_{4}$ is larger than $\delta /\left|x_{1}\right|$, the system enters the region $M^{+}$or $M^{-}$. This happens at about 10 seconds in Figure 4. The controller is switched to the exact feedback linearisation control law and the exact zero dynamics appear. As shown in Figure 5, the exact zero dynamics drive $x_{3}$ from 0 to $\pi$. As $x_{3}$ increases from $\pi / 2$ to $\pi, x_{4}$ decreases. Once the magnitude of $x_{4}$ is less than $\delta /\left|x_{1}\right|$, the controller is switched to the approximate linearisation control law. Since $\left(r_{0}, 0, \pi, 0\right)$ is another equilibrium under the approximate control law, the system moves around this equilibrium and $x_{3}$ varies around $\pi$ due to the driven internal dynamics. This is shown in Figure 4 between 12-25 seconds. Again once the magnitude of $x_{4}$ is larger than $\delta /\left|x_{1}\right|$ as $\left|x_{1}\right|$ increases, the exact linearisation control law is employed and the state is driven from $\pi$ to 0 . Then the above process is repeated. This is why the beam oscillates between $x_{3}=0$ and $x_{3}=\pi$.

In other words, the control scheme for the ball and beam example switches between a stable closed-loop system (when the state is in $M_{0}$ ) and an unstable closed-loop system (when the state is in $M^{+}$or $M^{-}$). If the ball and beam system is written in the format of (5), it is clearly seen that $\dot{x}=f^{\mathrm{ex}}\left(x, y_{D}\right)$ is unstable and $\dot{x}=f^{\text {app }}\left(x, y_{D}\right)$ is stable. To investigate the applicability of the switching control scheme for such a nonlinear system, first one should design a stabilising exact linearisation control law. For a nonlinear system with unstable zero dynamics as the ball and beam example, Devasia-Chen-Paden's nonlinear inversion 
technique [1] may be used to design such a control.

\section{Conclusions}

This paper considers and adds to the work of Tomlin and Sastry [4]. It further investigates the applicability of the switching control scheme proposed in [4] for nonlinear systems with ill-defined relative degree. It is shown that the switching control scheme is applicable not only for nonlinear systems with unchanged equilibria under the switching but also for nonlinear systems whose equilibria change. Therefore the switching feedback linearisation control scheme can be applied not only to Case 3 but also to Cases 1 and 2 in [4]. The results in this paper significantly extends the application range of this method. It is also pointed out that a necessary condition for the switching control scheme being applicable is that the region of approximate linearisation control laws is attractive to the exact zero dynamics.

\section{References}

[1] S. Devasia, D. Chen, and B. Paden. Nonlinear inversion-based output tracking. IEEE Trans. on Automatic Control, 41(7):930-942, 1996.

[2] J. Hauser, S. Sastry, and P.V. Kokotovic. Nonlinear control via approximate input-output linearization: the ball and beam example. IEEE Trans. on Automatic Control, 37(3):392-398, 1992.

[3] A. Isidori. Nonlinear Control Systems: An Introduction. 3rd Ed. Springer-Verlag, New York, 1995.

[4] C. J. Tomlin and S. S. Sastry. Switching through singularities. Systems \& Control Letter, $35: 145-154,1998$. 


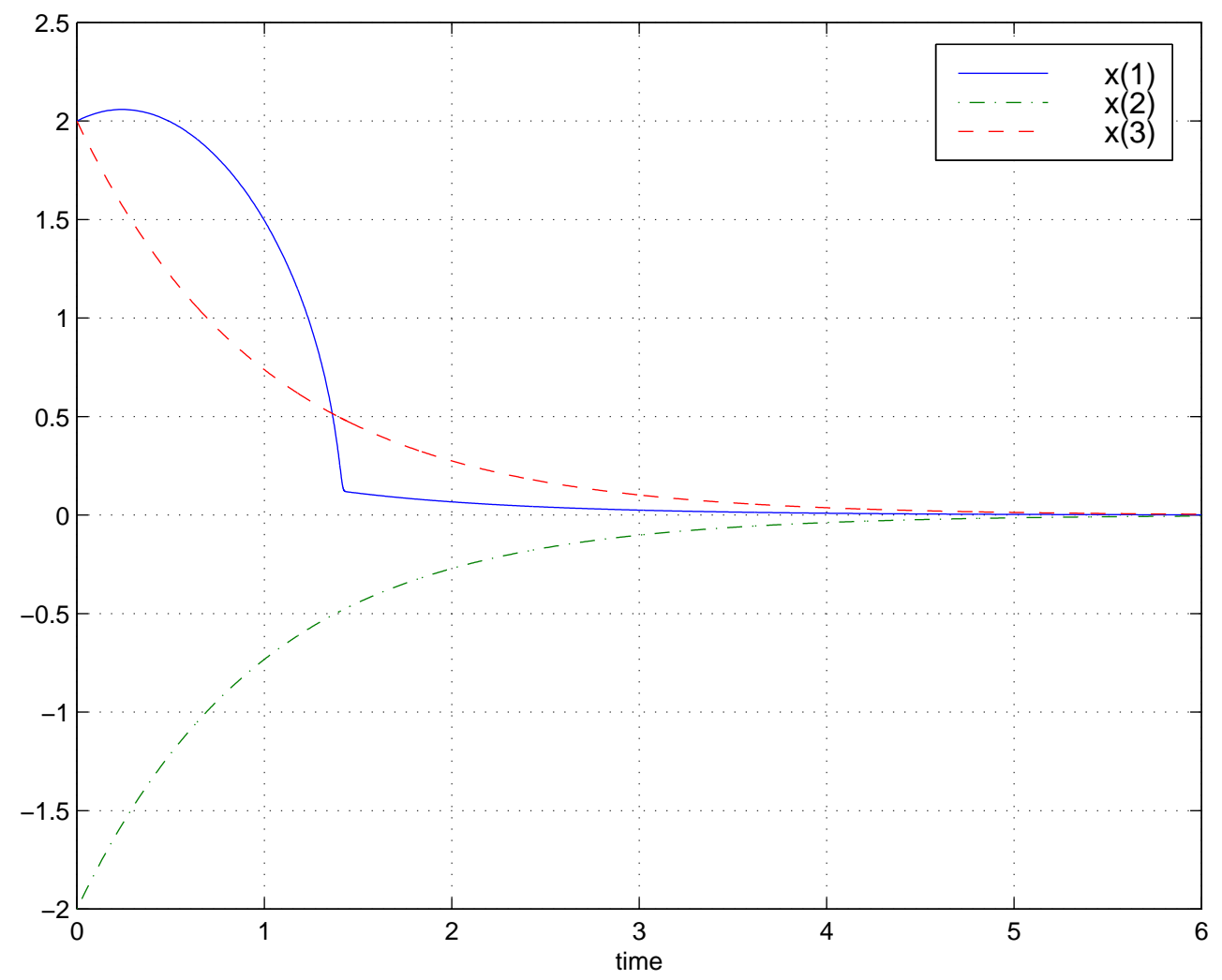

Fig. 1. The performance under the switching control: Case 1 


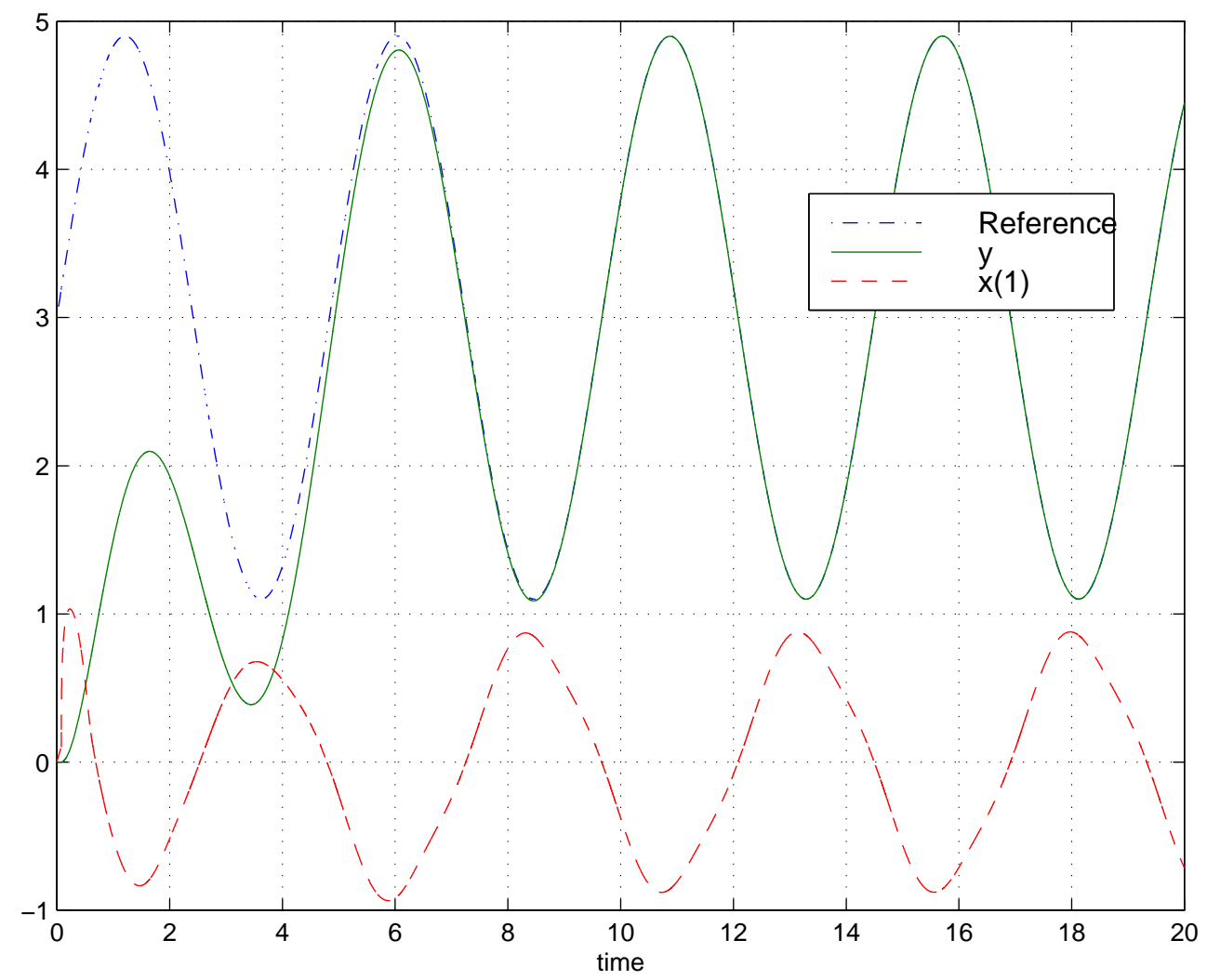

Fig. 2. The tracking performance under the switching control: Case 1 


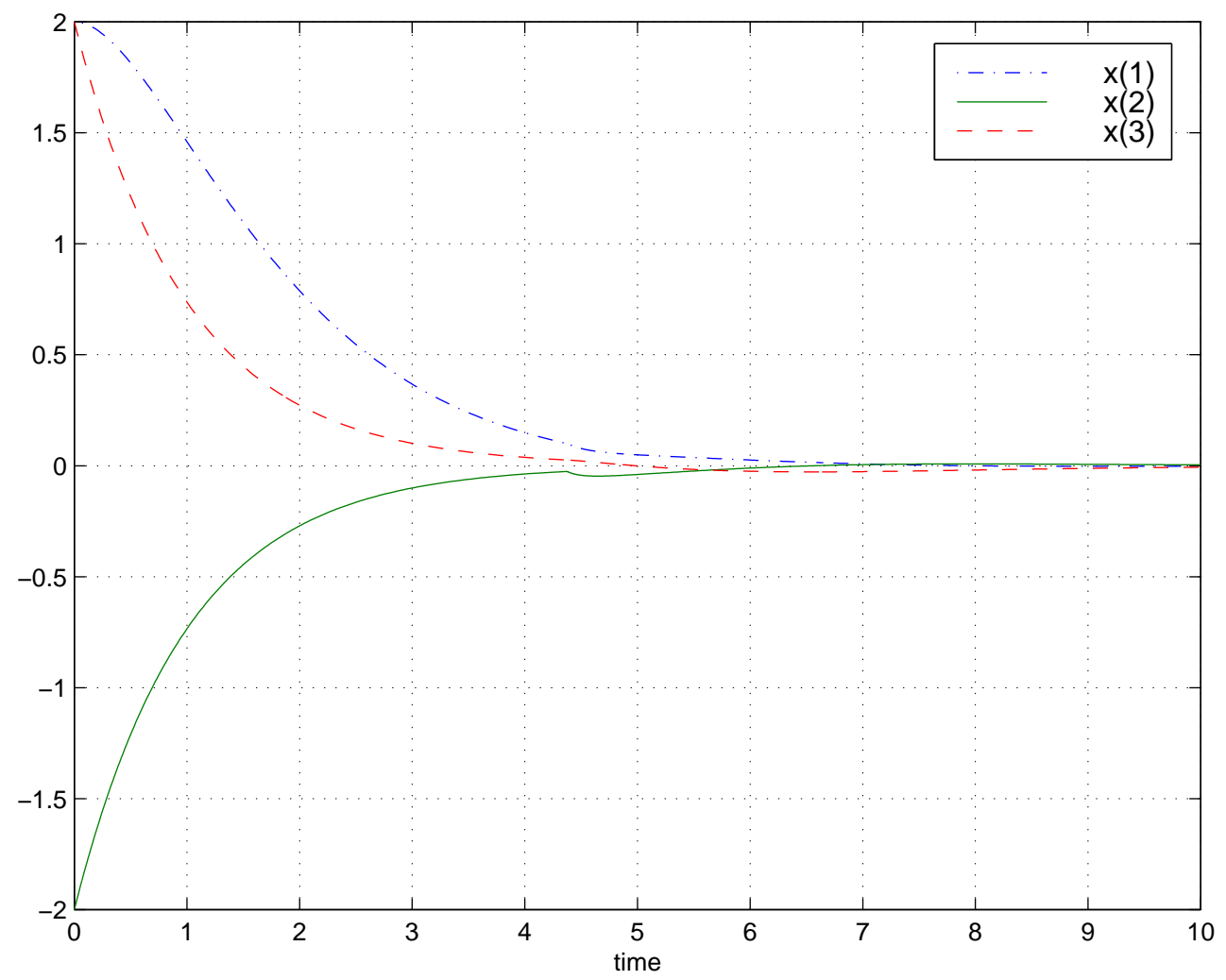

Fig. 3. The performance under the switching control: Case 2 


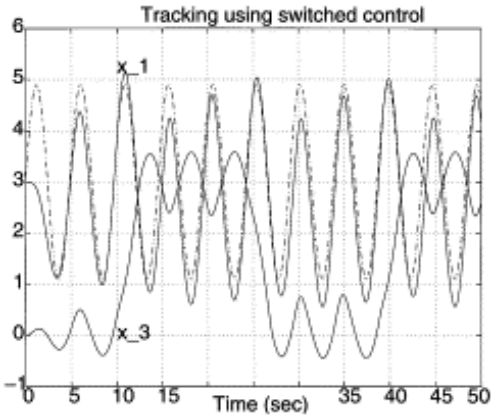

Fig. 4. Tracking performance of the ball and beam system under the switching control law, reproduced from Figure 2 in $[4]$ 


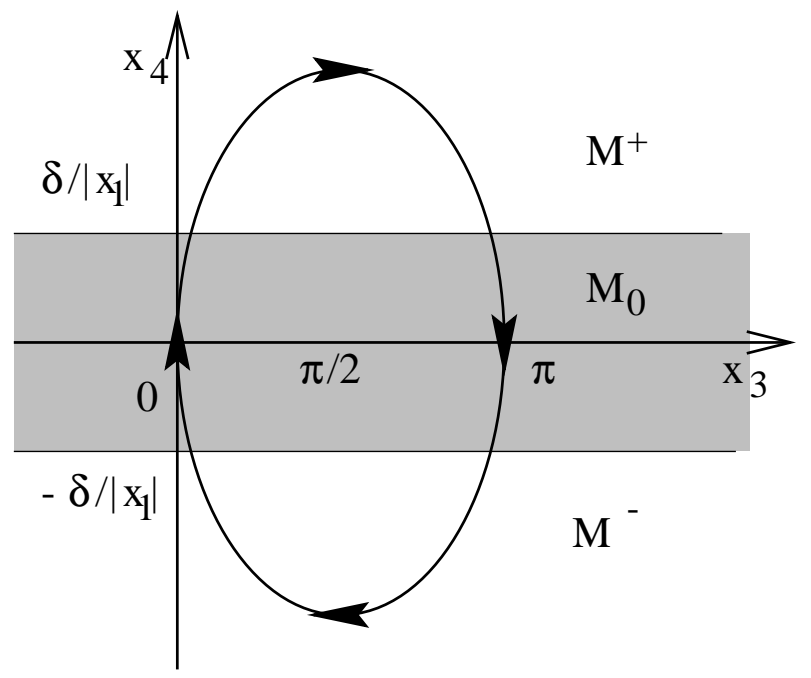

Fig. 5. The exact zero dynamics of the ball and beam example 